\title{
Letters to the Editor-August 17, 2020
}

\author{
Physicists returning to the lab after the long shutdown adjust to \\ lone-working protocols and appreciate some gains in productivity.
}

$\square$ o you have a story to share about your experience with labs re-opening during the pandemic? Write to physics@aps.org. A selection of letters will be posted in Physics and APS News.

\section{New Protocols and New Habits}

Our department re-opened in July but for essential laboratory work only. The re-opening was preceded by an intense month, during which we worked on new protocols to ensure everybody would be safe in the building, which proved to be quite tricky because of the constantly evolving knowledge of how the virus propagates. Are we doing enough or too much?

Making our laboratories COVID-19-safe was probably the easiest part. Indeed, these spaces consist mainly of individual clean rooms with huge air extraction mechanisms. People wear PPE while preparing their geological samples for isotope analyses. However, solo working, which was previously the exception rather than the rule, became a new concern because of the low building occupancy requirements. We had to set up a strict

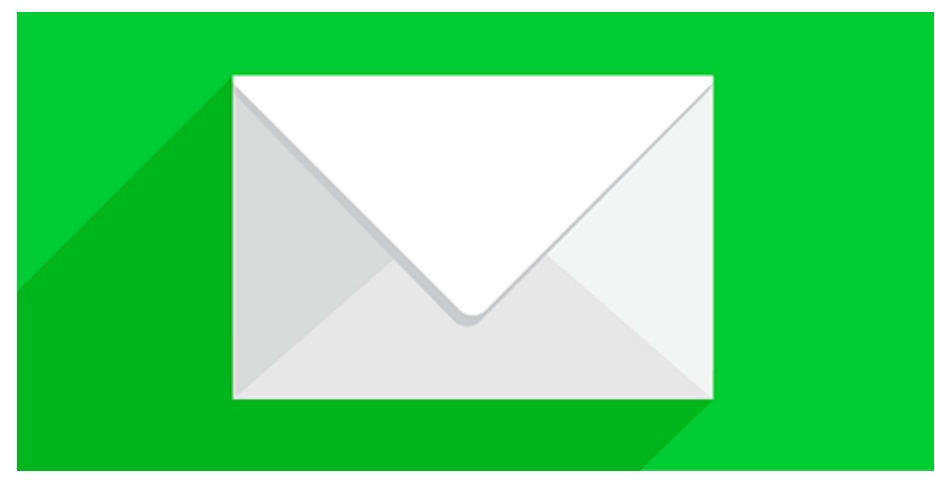

Credit: APS/Carin Cain "buddy" system for anyone who works directly with chemicals. Juggling between lab work and other activities has required adjustments because our offices are still mostly off-limits. But most of all, we've had to adjust to lab days that are not punctuated by informal interactions with our lab mates.

- Marie-Laure Bagard is the Laboratory Manager at the Department of Earth Sciences, University of Cambridge, UK.

\section{Prioritizing Publication and Repairing Equipment}

In the first week of June, research assistants, specialists, and professors returned to the lab. We prioritized people who needed immediate results for a reviewed paper or from a process that got disrupted because of the sudden shutdown. In July, we focused on completing work that was disrupted by the shutdown. After July, our graduate students joined us. But many foreign students have still not returned.

Social distancing rules strongly affect our High Magnetic Field \& Low-Temperature Lab. The experimental system usually requires a minimum of three people, so we've had to delay measurements. Work continues in the Characterization Lab and Advanced Lithography Lab, where social distancing rules are easy to satisfy. However, we do not have technicians to control, check, and repair instruments, so doing that consumes much of our time. Teams of researchers take turns managing the labs. Still, downtime means we have extra time to analyze data and write up our unpublished work, and we seem to be more productive.

- Ömer Dönmez is an Associate Professor of Applied Physics in the Natural Science Faculty at Istanbul University, Turkey. 


\section{Cautious and Controlled}

This dark time of the COVID-19 pandemic revealed the "everyday heroes" among us. Thanks to their efforts, the pandemic was controlled relatively quickly in China. In late March, faculty members at our university returned to the laboratories. Then, from May to June, graduate students were gradually allowed back in a very cautious and organized way. Fortunately, nobody at the university was affected by the virus. We have since been able to continue our research. During the lockdown, I spent quality time reading papers and books. The pandemic also triggered an interesting shift from constant travel to online meetings, which brings several benefits, such as saving time and money and avoiding personal disruption. Thanks to the internet, our group meetings and journal club never stopped.

- Chao-Yang Lu is a Professor of Physics at the University of Science and Technology of China, Hefei.

\section{Supercomputers and Superior Support}

My research group has largely been unaffected by the shutdown. However, we were well prepared for remote work because we spent time on-site at the Environmental Molecular Sciences Laboratory (EMSL) in February, analyzing organic particles that accumulate in the atmosphere. During our visit we understood very well what we could expect from the EMSL support staff, and they understood what complexities exist within our research. Perhaps we could have reached such understanding remotely, but I believe the research benefited from our in-person interactions. We've been working on mass spectrometry data that we acquired in February, and we continue to run atmospheric model simulations on an EMSL supercomputer.

- Lynn Mazzoleni is an Associate Professor in the Department of Chemistry at Michigan Technical University.

\section{Careful Scheduling and Solo Work}

The pandemic has had a substantial effect on how we go about our spectroscopy work on quantum materials. Along with an overall reduction in the number of students and employees on campus, labs are limited to one individual per room. This need requires scheduling and coordination of lab activities ahead of time, taking every precaution to avoid accidental overlap of researchers in small spaces. We wear masks, routinely disinfect shared surfaces, and prepare for the possibility that we may need to change our behavior at a moment's notice. Since fewer people are in the lab each day, we increase productivity on other work that can be done elsewhere; for example, data analysis from previous experiments, manuscript preparation, writing proposals, or smaller solo experimental tasks.

- Adam Gross is a Ph.D. student in the Department of Physics at the University of California, Davis.

\section{Homeward Bound, Again}

We had just commenced a new semester when the first COVID-19 case was reported in Nigeria. All the students were sent home, and the faculty developed a plan for online teaching. After adopting some safety protocols, the university asked graduate students to return for teaching in large lecture halls where social distancing and mask wearing became the norm. But as case numbers began to increase nationally, those students were asked to return home again.

Now, I continue work with my graduate and undergraduate students on projects conducted from home and supervised via teleconferencing. In "Home/Fun Physics," students use items they can find at home as apparatus for various experiments. Not only are they able to learn from the experiments, but they realize that physics itself is an everyday experience. The students use smartphones as measuring devices for these experiments. Finally, my students use QPython as a mobile computational laboratory for learning programming anywhere. I have been having a very engaging and exciting period during this pandemic.

- Godfrey Akpojotor is a Professor of Theoretical Physics and the Director of Research and International Programs at the Delta State University, Abraka, Nigeria. 